\section{Gene puffs}

GENE therapists exercise their skills with high-tech tools borrowed from viruses to correct faulty genes in celis. A different approach to the same end is described by R. Stribling et al., who used cationic liposomes to wrap up a prokaryotic gene for direct delivery by aerosol into the lungs of mice (Proc. natn. Acad. Sci. U.S.A. 89, 11277-11281; 1992). The bacterial protein went on being expressed in all the conducting airways of the lung for more than three weeks after the gene encoding it was inhaled, apparently without damage to the mouse. Although any effects of repeatedly sniffing these high-fat DNA particles still need to be determined, the authors are optimistic that their technique may one day be used to help sufferers of cystic fibrosis.

\section{Censor's triumph}

Cosmic censorship, the hypothesis formulated to allow general relativists to sleep at night, has it that any gravitational singularity must always be concealed behind an event horizon. Whether this is invariably true is an unanswered question, and some relativists, eager to disturb their colleagues, have tried to construct 'naked' singularities - unsuccessfully so far. A. Abrahams and C. Evans (Phys. Rev. D46, R4117-R4121; 1992) have also tried to evade the cosmic censor. By careful arrangement of initial conditions, they have numerically calculated the evolution of an imploding gravitational wave with axisymmetric (quadrupole) symmetry. The energy in the wave converges on a point, increasing the local density without limit so that a singularity forms. But, as always, an event horizon forms first, and the singularity is hidden. What

Abrahams and Evans have found, instead, is an extremely difficult way to make a black hole.

\section{Waste management}

Do pea aphids, Acyrthosiphon pisum, get by with a little help from their symbiotic bacterial friends in recycling aphid waste ammonia to essential amino acids? Yes (or probably yes) conclude $\mathrm{L}$. F. Whitehead et al. (Proc. R. Soc. B250, $115-117 ; 1992)$. The authors took two approaches. Bacteria isolated from the aphid can accumulate methylamine, an analogue of ammonia; and if in some unhappy aphids the bacteria are disrupted by antibiotics, the ammonia content in their excreta rises 3-4 times. Whitehead et al. tie these results into work showing that bacteria release amino acids essential for the aphids. But the conclusion remains tentative: it is not entirely certain that methylamine and ammonia share the same transport system in bacteria, or that something other than the bacteria is not perturbed by the antibiotics.

\title{
A tale of two families
}

\section{Susan G. Amara}

MAJOR advances in the cloning of neurotransmitter transporters over the past three years make this the best of times to examine how these high-affinity reuptake systems terminate synaptic transmission. The notable exceptions to this cloning onslaught have been the eagerly anticipated DNA sequences encoding the glutamate transporters, the glial and neuronal uptake systems that sequester glutamate released at the most abundant class of excitatory synapses. The reason for this curious omission is now evident from three papers, one in the Proceedings of the National Academy of Sciences, and two appearing on pages 464 and 467 of this issue: glutamate transport is accomplished by a multigene family distinct from the gene family that includes all other neurotransmitter transporters isolated so $\mathrm{far}^{1-3}$.

The molecular characterization of neurotransmitter carriers began with the purification, amino-acid sequencing and cloning of a $\gamma$-aminobutyric acid (GABA) transporter, and was consolidated when the expression cloning of the noradrenaline transporter demonstrated that a transporter gene family exists. Since then, a growing list of neurotransmitter transporters have been isolated by methods that rely on the sequence conservation within the 12 transmembrane structural motifs of this gene family ${ }^{4}$. Members of the family, which includes transporters for the biogenic amines, GABA, glycine, choline, proline and taurine, all seem to link substrate influx to the cotransport of $\mathrm{Na}^{+}$and $\mathrm{Cl}^{-}$ions across the plasma membrane. Glutamate transport, however, has appeared mechanistically distinct because amino-acid influx is coupled to the cotransport of $\mathrm{Na}^{+}$and the countertransport of $\mathrm{K}^{+}$, with no dependence on $\mathrm{Cl}^{-}$. Such functional distinctions are now explained by the demonstration that glutamate transporters represent a very different structural class of amino-acid carriers.

Three distinct complementary DNAs encoding structurally related excitatory amino-acid transporters have now been isolated by returning to methods that do not rely on family relationships. Serendipity led Storck et al. ${ }^{1}$ to a glutamate/ aspartate transporter cDNA, referred to as GLAST 1, which was identified using oligonucleotide probes based on the sequence of a protein that co-purified with a ceramide galactosyl transferase. The RNA encoding the transporter is specifically expressed in the brain, and although its precise cellular location has not been determined its diffuse distribution and properties may be more con- sistent with those of a glial glutamate transporter. Pines et $a$ l. $^{2}$ purified a protein with glutamate transport activity from rat brain and used the protein to obtain antibodies for screening a bacteriophage cDNA expression library. The resulting cDNA clone, referred to as GLT-1, detects an RNA species in rat brain, but not in any peripheral tissues tested, and in a separate report the authors provide evidence that, like GLAST 1, the GLT-1 gene product is expressed predominantly in glial cells 5 .

Using a different approach, Kanai and Hediger $^{3}$ screened a plasmid cDNA library for expression of high-affinity uptake activity in Xenopus oocytes to obtain a cDNA which they term EAAC1. In situ hybridization suggests that the expression of EAAC1 within the central nervous system is largely neuronal, and it includes expression within the hotbed of glutamatergic synapse research, the pyramidal cells of the hippocampus. Neurobiologists may find the distribution of the EAAC1 gene product rather surprising, because although the high-affinity transporter is expressed abundantly in the central nervous system, it is also found in several peripheral tissues and was actually identified in a cDNA library from rabbit small intestine.

GLAST 1, GLT-1 and EAAC1 are very similar in their amino-acid sequences ( $\sim 50 \%$ identical) and in their proposed structural characteristics, establishing these genes as members of a new family. Surprisingly, database searches for related sequences by all three groups found significant homology with the proton-coupled glutamate transporter glt-P of Escherichia coli $^{6}$, as well as several other prokaryotic glutamate and dicarboxylate transporters. Particularly striking is the conservation of the sevenamino-acid sequence AAIFIAQ found both in the $E$. coli and mammalian proteins, which suggests a rather longstanding familial relationship. The proposed membrane topology of these glutamate transporters is somewhat ambiguous, given the subjective application of structural algorithms. There is general agreement on the location of the first six transmembrane domains in the three transporters, but the laboratories differ on the total number of membrane-spanning segments, proposing 6,8 or 10 . In the light of such controversy, perhaps it is better to distinguish the two plasma membrane transporter gene families not by the number of transmembrane domains (12 versus $6-10$ ), but by their ionic depend- 